\title{
A Solution Approach from an Analytic Model to Heuristic Algorithm for Special Case of Vehicle Routing Problem with Stochastic Demands
}

\author{
Selçuk K. İşleyen ${ }^{1}$ and Ö. Faruk Baykoç² \\ ${ }^{1}$ Department of Industrial Engineering, Ataturk University, 25240 Erzurum, Turkey \\ ${ }^{2}$ Department of Industrial Engineering, Gazi University, Maltepe, 06570 Ankara, Turkey \\ Correspondence should be addressed to Selçuk K. İşleyen, isleyens@gazi.edu.tr
}

Received 18 August 2008; Accepted 11 December 2008

Recommended by Irina Trendafilova

\begin{abstract}
We define a special case for the vehicle routing problem with stochastic demands (SC-VRPSD) where customer demands are normally distributed. We propose a new linear model for computing the expected length of a tour in SC-VRPSD. The proposed model is based on the integration of the "Traveling Salesman Problem" (TSP) and the Assignment Problem. For large-scale problems, we also use an Iterated Local Search (ILS) algorithm in order to reach an effective solution.

Copyright (C) 2008 S. K. İşleyen and Ö. F. Baykoç. This is an open access article distributed under the Creative Commons Attribution License, which permits unrestricted use, distribution, and reproduction in any medium, provided the original work is properly cited.
\end{abstract}

\section{Introduction}

The classical Vehicle Routing Problem (VRP) is often defined as serving customers from a central depot with a fleet of vehicles, each having a limited capacity. The objective of the problem is to minimize either total transportation costs or total distance traveled. Each vehicle must follow a valid initial tour and ending at the depot, and each customer must be visited exactly once. The total service level required at the customer locations on the tour may not exceed the capacity of the assigned vehicle. The classical VRP is an important problem in the field of logistics and distribution. See Laporte and Osman [1], Toth and Vigo [2], Laporte et al. [3], Tarantilis et al. [4] for more detailed studies of the deterministic VRP and its extensions.

In the deterministic VRP, it is assumed that travel times, customer demands and cases of customers' existence are pre-determined. However, in real-life problems, one or more of these parameters may not be precisely defined. Problem types occurring in such situations are defined in the literature as a Stochastic Vehicle Routing Problem (SVRP). In the SVRPs, customer sets that will be visited, customers' demands or travel times are modeled as random variables derived from a known probability distribution. 
Gendreau et al. [5] analyzed SVRP in three categories: the Vehicle Routing Problem with Stochastic Demand (VRPSD), VRP with Stochastic Customers (VRPSC), and VRP with Stochastic Customers and Demands (VRPSCD).

This paper considers the capacity-constrained vehicle routing problem with stochastic demand (VRPSD), where only the customer demand is stochastic and all other parameters are pre-determined. This problem appears in many practical situations, and prior applications include the delivery of home heating oil [6], cash collection from bank branches [7] and sludge disposal [8].

In VRPSD problems, a vehicle with a finite capacity (termed $Q$ ) leaves the depot fully loaded and services the set of customers whose demands are established only after reaching the customer location. The planned route starts from the depot and ends by returning to the depot after visiting each customer at least once. The planned route is called an "a priori tour". An a priori tour identifies which customers at which ranks will be serviced. However, the real route also includes returns to the depot when required (as when reloading the vehicle). In some cases, the vehicle may thus be unable to satisfy the customer's demand when the actual demand along the route exceeds the vehicle's capacity. Such a situation is referred to as a route failure. The vehicle routing problem with stochastic demands (VRPSD) consists of minimizing the total cost of the planned routes and expected failures. To ensure feasibility of solutions in case of route failure, recourse policies or corrective actions have to be designed.

Generally, there are three types of recourse policies used for studies. The first, known as a simple recourse policy, states that when a route failure occurs (when its' capacity is exceeded), a vehicle returns to the depot, reloads and continues its tour by returning to the node where the failure occurred $[5,9]$. The second policy is termed a preventive restocking policy. In this approach, before route failure occurs, instead of proceeding to the next customer, the vehicle returns to the depot according to the remaining load quantity and the location of the customer [10-12]. The third policy type, developed by Secomandi $[13,14]$ applies neuro-dynamic programming techniques to VRPSD. The basis of this approach is that, after the customer demands are known or, after each failure, the remaining portion of the a priori tour must be optimized again, rather than completed as originally planned. While this approach is able to provide outputs having smaller expected values than the preventive stocking strategy, it is relatively difficult to compute.

In this paper, the Vehicle Routing Problem with Stochastic Demands (VRPSD) is considered where customer demands are independent and identically distributed -each customer demand is normally distributed and has the same mean and standard deviation. This situation is referred to as the Special Case of the VRPSD (SC-VRPSD). The Special Case scenario also presumes that the service policy is non-divisible, meaning that the entire demand at each customer must be served in a single visit by a unique vehicle. In case of route failure, the first (simple recourse) policy is used as a recourse action. The present study proposes a new integer mathematical model for efficiently computing the expected length of a tour. The methodology applies an Iterated Local Search (ILS) to SC-VRPSD problems which are too large to be solved by the proposed mathematical model.

The rest of the paper is organized as follows; in the following section, the definition and some studies related to the VRPSD problem are summarized. Section 3 investigates how to calculate the expected cost of an a priori tour. In Section 4, the Special Case for VRPSD is examined and a linear mathematical model is established for SC-VRPSDs. In Section 5, some Traveling Salesman Problems (TSP) which are well known in the literature were converted into SC-VRSPD problems and solutions were sought for several vehicle capacities by using ILS. Section 6 presents conclusions and suggestions for future research. 


\section{Formal description for VRPSD}

The VRPSD problem is defined on a complete graph $G=(V, A, D)$, where $V=\{0,1, \ldots, n\}$ is the set of nodes (customers). While node 0 represents the depot, $A=\{(i, j): i \neq j, i, j \in V\}$ is the set of arcs conjoining the nodes and $D=\left\{d_{i j}: i \neq j, i, j \in V\right\}$ is the travel times or distances between the nodes. The distance matrix $D$ is symmetrical and provides triangular inequality: $d(i, j) \leq d(i, k)+d(k, j)$. The positive integer $Q$ denotes the vehicle capacity. The present study considers only a single vehicle. While a vehicle with capacity $Q$ is providing service according to the customer demands, the total expected travel distance is also minimized. If vehicle capacity is exceeded during service, the vehicle returns to the depot to be restocked to the capacity $Q$. When all demands have been served, the vehicle returns to the depot. The following assumptions are made in VRPSD problems.

(i) Customer demands $\left(\xi_{i},\right)$ are stochastic variables independently distributed with known distributions $\xi_{i}, i=1, \ldots, n$.

(ii) The real demand of each customer is only known when the vehicle reaches them.

(iii) Customer demands $\xi_{i}$ cannot exceed the vehicle capacity $Q$ and the demands may be derived from the discrete or continuous probability distributions.

A feasible solution to the VRPSD is a permutation of the customers $s=(s(0), s(1), \ldots$, $s(n), s(0)),(s(0)=0)$, and it is called an a priori tour. The vehicle visits the customers in the order given by the a priori tour. The objective function to be minimized is the expected cost of the a priori tour.

Gendreau et al. [15] presented an exact stochastic integer programming method for VRP scenarios with both stochastic customers and stochastic demand (VRPSCD) (integer Lshaped method). The same method has also been applied to VRPSs having only stochastic demand (VRPSD). In the problems with both stochastic customers and demands (VRPSCD), they presented solutions for scenarios with up to 46 nodes and, in the problems having only stochastic demands (VRPSD), they presented solutions for scenarios with up to 70 nodes and two vehicles. The same researchers [16] have developed a Tabu search algorithm (TABUSTOCH) for problems too large to be solved by the L-shaped method.

Teodorović and Pavković [17] proposed a Simulated Annealing algorithm for the solution of VRPSD with multiple vehicles. This model permitted a maximum of one failure on each route.

Isleyen and Baykoc [18] (article in press) suggested a model which effectively calculated the expected cost of an a priori tour given for a vehicle routing problem with stochastic demand in which the demands were normally distributed. They used a MonteCarlo Simulation for the determination of the correctness of their model.

Yang et al. [11] analyzed the VRPSD with single and multiple vehicles. They assumed that the expected distance traveled by each vehicle cannot exceed a certain value. Researchers have tested two heuristic algorithms, route-first/cluster-next and cluster-first/route-next. These algorithms have been used to define sets of customers to be serviced by different vehicles, and then to find the optimal route for each customer set. Both algorithms have worked effectively for small problems involving up to 15 customers.

Bianchi et al. [12] analyzed the performance of meta-heuristics for solving VRPSDs with discrete demand. Because of their computational ease, the researchers used the "traveling salesman problem" (TSP) approach and Or-opt operations to compute the objective function. Using these techniques, the researchers evaluated the performance of 


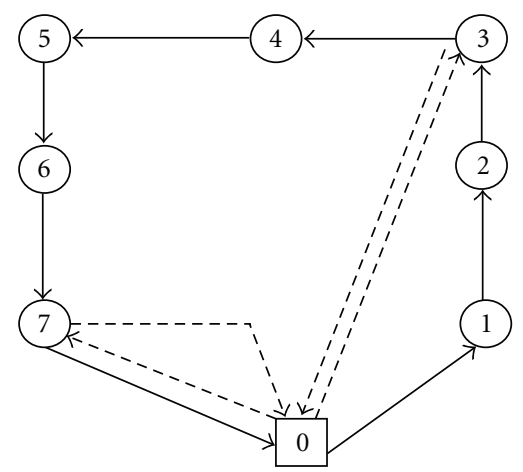

Figure 1: Service policy of the vehicle.

the following metaheuristics: Iterated Local Search, Tabu Search, Simulated Annealing, Ant Colony Optimization, and Evolutionary Algorithm.

Dror and Trudeau [19] developed a model for computing the expected distance of the a priori tour. However, their work considered only one failure and they assumed that service must be given to customers individually for nodes at which route failure has occurred as well as for the subsequent nodes.

Other approaches have been taken in the detailed studies of Bertsimas and Simchi-Levi [20] and Kenyon and Morton [21].

The most difficult and the most important part of the VRPSD problem is to calculate the expected cost of an a priori tour. In the following section, the expected cost will be calculated for a VRPSD problem in which customer demands are normally distributed.

\section{Expected cost of the a priori tour}

In most VRPSD studies, stochastic demands are derived from a discrete probability distribution. The current study differs from those in the existing literature in a number of ways. In the present study, a continuous normal distribution is used rather than a discrete distribution. In addition, the current model analyzes multiple failures that may occur on the single route, which are included in computation of the expected tour length. Furthermore, the current model assumes that the service policy is non-divisible, meaning that each customer's entire demand must be served in a single visit by a unique vehicle.

To illustrate the service policy, consider the following example. The a priori tour is $(0,1,2,3,4,5,6,7,0)$ where node 0 is the depot; the vehicle has a capacity of 20 and the realization of the customer demands are $\xi_{1}=10, \xi_{2}=8, \xi_{3}=6, \xi_{4}=7, \xi_{5}=3, \xi_{6}=4$, and $\xi_{7}=5$. Under the service policy that the demands are not divisible, the resulting routes are shown in Figure 1. Note that at nodes 3 and 7 the vehicle capacity is exceeded and the vehicle is forced to return to the depot. Thus, the length of the a priori tour is increased by the addition of the distances due to the route failures at these two nodes.

Notations

$d_{i, j}=$ the distance between customer $i$ and $j$ during the route;

$\xi_{i}, i=1, \ldots, n$ (Stochastic demands) independent random variable $\xi_{i}$, that is, normally distributed with a finite mean $\mu_{i}$ and finite standard deviation $\sigma_{i}$; 
$Q=$ vehicle capacity;

$p_{i, j}$ : Probability of meeting the demands of the customers from node $i$ to node $j$ on a given a priori tour ( $i$ and $j$ inclusive);

$C_{i}$ : total route failure probability of customers in the $i$ th service order;

$T_{i}$ : the length of additional distance stemming from the route failure in the $i$ th service order;

If an a priori route is specified, the $s_{i}=\left(s(0), s_{1}(r), \ldots, s_{n}(r), s(0)\right)$;

$L$ : length of the a priori tour (TSP length);

$$
\begin{gathered}
L=\sum_{i=1}^{n-1} d_{s_{i}(r), s_{i+1}(r)}+d_{s(0), s_{1}(r)}+d_{s_{n}(r), s(0),} \\
C_{i}=\sum_{j=1}^{i-1}\left(p_{j, i-1}-p_{j, i}\right) * C_{j} \quad i=2,3, \ldots, n, \\
T_{i}=C_{i} * 2 * d_{0, i} \quad i=2,3, \ldots, n .
\end{gathered}
$$

With the boundary condition

$$
C_{1}=1
$$

And it is assumed that $\xi_{i}$ does not exceed the vehicle capacity $Q\left(p_{i, i}=1, i=1, \ldots, n\right)$.

Total cost stemming from route failure $\sum_{i=2}^{n} T_{i}$.

Finally, the expected length of the a priori tour is $L+\sum_{i=2}^{n} T_{i}$.

In the model, $p_{j, i-1}-p_{j, i}$ is the probability of meeting the demands of customers from node $j$ to the node $i-1$ (including $j$ and $i-1$ ) and not meeting the demand of the $i$ th customer.

If a failure occurs in any node $i$, the extra distance that will be traveled by the vehicle is $2 d_{0 i}$ which means traveling from the node to the depot and then returning to the same node to resume the tour.

When non-failure probabilities are computed, the summation ability of the normal distribution and standard normal distribution are used, as shown:

$$
\begin{gathered}
\xi_{1} \sim N\left(\mu_{1}, \sigma_{1}\right), \\
\xi_{2} \sim N\left(\mu_{2}, \sigma_{2}\right), \\
\xi_{1}+\xi_{2} \sim N\left(\mu_{1}+\mu_{2}, \sqrt{\sigma_{1}^{2}+\sigma_{2}^{2}}\right), \\
p_{12}=P\left[\left(Q-\left(\xi_{1}+\xi_{2}\right)\right) \geq 0\right], \\
p_{13}=P\left[\left(Q-\left(\xi_{1}+\xi_{2}+\xi_{3}\right)\right) \geq 0\right], \\
\vdots \\
p_{n-1, n}=P\left[\left(Q-\left(\xi_{n-1}+\xi_{n}\right)\right) \geq 0\right] .
\end{gathered}
$$


Table 1: Dataset of sample problem.

\begin{tabular}{lcccc}
\hline Customers & x_coord & y_coord & Average demand & stdv \\
\hline 0 & 1 & 1 & 0 & 0 \\
1 & 67 & 80 & 50 & 10 \\
2 & 72 & 20 & 46 & 9.2 \\
3 & 49 & 56 & 20 & 4 \\
4 & 83 & 97 & 31 & 6.2 \\
5 & 82 & 30 & 43 & 8.6 \\
6 & 73 & 58 & 28 & 5.6 \\
7 & 4 & 5 & 43 & 8.6 \\
8 & 70 & 51 & 39 & 7.8 \\
& & & Total demand $=300$ & Vehicle capacity $=150$ \\
\hline
\end{tabular}

Table 2: Probabilities of meeting the demands.

\begin{tabular}{lllllll}
\hline$p 12=0.9999$ & $p 23=1$ & $p 34=1$ & $p 45=0.9999$ & $p 56=0.9999$ & $p 67=0.9999$ & $p 78=0.9999$ \\
$p 13=0.9918$ & $p 24=0.9999$ & $p 35=0.9999$ & $p 46=0.9999$ & $p 57=0.9964$ & $p 68=0.9990$ & \\
$p 14=0.5769$ & $p 25=0.7534$ & $p 36=0.9866$ & $p 47=0.6326$ & $p 58=0.4232$ & & \\
$p 15=0.0119$ & $p 26=0.1248$ & $p 37=0.1633$ & $p 48=0.0208$ & & & \\
$p 16=0.0001$ & $p 27=0.0003$ & $p 38=0.0008$ & & & & \\
$p 17=2.8678 \mathrm{E}-08$ & $p 28=1.4085 \mathrm{E}-07$ & & & & & \\
$p 18=3.6350 \mathrm{E}-12$ & & & & & & \\
\hline
\end{tabular}

If we generalize;

$$
p_{i, j}=P\left[\left(Q-\sum_{l=i}^{j} \xi_{l}\right) \geq 0\right] .
$$

If we say $\sum_{l=i}^{j} \xi_{l}=X$

$$
\begin{gathered}
X \sim N\left(\mu_{X}, \sigma_{X}\right), \\
P(X \leq Q)=P\left(\frac{X-\mu_{X}}{\sigma_{X}}<\frac{Q-\mu_{X}}{\sigma_{X}}\right), \\
P(X \leq Q)=P\left(Z<\frac{Q-\mu_{X}}{\sigma_{X}}\right) .
\end{gathered}
$$

From (3.7), nonfailure probabilities are computed.

Numerical example

A test problem with eight customers was generated in order to explain the model. Data for the problem are given in Table 1. Distances between the customers (whose locations are represented by $x-y$ coordinates) were rounded to the nearest integer.

The probability of meeting customer demands for an a priori tour (route 0-1-2-3-4-5-67-8-0) is shown in Table 2. 
Table 3: Route failure probabilities for 0-1-2-3-4-5-6-7-8-0 tour.

\begin{tabular}{|c|c|c|c|c|c|c|c|c|c|c|}
\hline Service order & & 1 & 2 & 3 & 4 & 5 & 6 & 7 & 8 & \\
\hline Initial tour & 0 & 1 & 2 & 3 & 4 & 5 & 6 & 7 & 8 & 0 \\
\hline$C_{i}$ & & 1 & 1E-04 & 0.00815 & 0.41488 & 0.565 & 0.0119 & 0.1613 & 0.579 & \\
\hline$d_{0 i}$ & & & 73 & 73 & 126 & 86 & 92 & 5 & 85 & \\
\hline$T_{i}$ & & & 0.0146 & 1.1899 & 104.5497 & 97.18 & 2.19 & 1.613 & 98.43 & \\
\hline
\end{tabular}

The length (L) of the 0-1-2-3-4-5-6-7-8-0 tour is 607 units (minimum possible distance, not including additional costs arising from route-failures).

It holds that

$$
\begin{gathered}
C_{1}=1, \\
C_{2}=p_{11}-p_{12}=1 e-4 \\
C_{3}=\left(p_{12}-p_{13}\right) * C_{1}+\left(p_{22}-p_{23}\right) * C_{2}=0.00815 \\
C_{4}=\left(p_{13}-p_{14}\right) * C_{1}+\left(p_{23}-p_{24}\right) * C_{2}+\left(p_{33}-p_{34}\right) * C_{3}=0.414889 .
\end{gathered}
$$

Other route failure probabilities are shown in Table 3.

$$
\sum T_{i}=305.175
$$

The total expected length of tour (including additional costs arising from route-failures) is $=607+305.175=912.175$.

The probability of meeting demands in the VRPSD scenario depends on the given initial tour. That means any change occurring in the a priori tour necessitates recalculating the probability of meeting demands for the partial set (the remaining customers) However, the present scenario determines that the demands of each customer have the same mean and standard deviation. Examining the normal distribution, it will be clear that probabilities of route failures in the service order will also be the same. In the next section, this special case for VRPSD will be examined and a linear mathematical model for SC-VRPSD will be established.

\section{Special case of vehicle routing problem with stochastic demands (SC-VRPSD)}

In the special case, the demands of each customer are assumed to be normally distributed, and the mean and standard deviation of the stochastic demands are assumed to be same for each customer.

$$
\xi_{i}=N(\mu, \sigma) \quad \forall_{i}
$$

The most remarkable characteristic of the special case is that the failure probabilities in the service order are always the same, In other words, failure probabilities are independent of the a priori tour.

Consider the SC-VRPSD with three customers, $i, j$, and $k$. Suppose all customers demands are normally distributed and have the same mean and standard deviation and the vehicle capacity is $Q$. 
If a priori tour $\mathrm{T} 1=0-i-j-k-0$ and the other priori tour $\mathrm{T} 2=0-j-k-i-0$

$$
\begin{aligned}
& \xi_{i}=N(\mu, \sigma), \quad \xi_{j}=N(\mu, \sigma), \quad \xi_{k}=N(\mu, \sigma), \\
& \xi_{r}=\text { the demand of the customer in the } r \text { th service order. }
\end{aligned}
$$

Service order $r r+1 r+2$

Tour $1(0-i-j-k-0) \quad i \quad j \quad k$

Tour $2(0-j-k-i-0) \quad j \quad k \quad i$

There are 3 nonfailure probabilities Tour 1 :

$$
\begin{gathered}
p_{i, j}=P\left[\left(Q-\sum_{l=i}^{j} \xi_{l}\right) \geq 0\right], \\
p_{r, r+1}=P\left[\left(Q-\sum_{l=r}^{r+1} \xi_{l}\right) \geq 0\right], \\
\xi_{r}+\xi_{r+1}=N \sim\left(\mu+\mu, \sqrt{\sigma^{2}+\sigma^{2}}\right)=N \sim(2 \mu, \sqrt{2} \sigma), \\
p_{r, r+1}=P[(Q-[N \sim(2 \mu, \sqrt{2} \sigma)) \geq 0](\mathrm{T} 1), \\
p_{r, r+2}=P[(Q-[N \sim(3 \mu, \sqrt{3} \sigma)]) \geq 0](\mathrm{T} 1), \\
p_{r+1, r+2}=P[(Q-[N \sim(2 \mu, \sqrt{2} \sigma)]) \geq 0](\mathrm{T} 1) .
\end{gathered}
$$

Similarly, nonfailure probabilities for Tour 2:

$$
\begin{gathered}
p_{r, r+1}=P[(Q-[N \sim(2 \mu, \sqrt{2} \sigma)]) \geq 0](\mathrm{T} 2), \\
p_{r, r+2}=P[(Q-[N \sim(3 \mu, \sqrt{3} \sigma)]) \geq 0](\mathrm{T} 2), \\
p_{r+1, r+2}=P[(Q-[N \sim(2 \mu, \sqrt{2} \sigma)]) \geq 0](\mathrm{T} 2) .
\end{gathered}
$$

As a result,

$$
\begin{aligned}
\mathrm{T} 1\left(p_{r, r+1}\right) & =\mathrm{T} 2\left(p_{r, r+1}\right), \\
\mathrm{T} 1\left(p_{r, r+2}\right) & =\mathrm{T} 2\left(p_{r, r+2}\right), \\
\mathrm{T} 1\left(p_{r+1, r+2}\right) & =\mathrm{T} 2\left(p_{r+1, r+2}\right) .
\end{aligned}
$$

It is suggested that failure probabilities are independent from the initial tour.

For the problem with 8 customers, previously outlined in Section 3, if we assume that all demands are $\xi_{i}=N(\mu, \sigma) \forall_{i}$ and $\sigma / \mu=0.2$ and the vehicle capacity is $4 \mu$.

The length $(\mathrm{L})$ of the $(0-1-2-3-4-5-6-7-8-0)$ a priori route $=607$ units (based on the a priori route and not including any un-planned returns to the depot to re-stock). In Table 4, route failure probabilities and the length of additional distances stemming from the route 
Table 4: Route failure probabilities and additional distances for a priori tour 0-1-2-3-4-5-6-7-8-0.

\begin{tabular}{lcccccccccc}
\hline Service order & & 1 & 2 & 3 & 4 & 5 & 6 & 7 & 8 \\
A priori tour & 0 & 1 & 2 & 3 & 4 & 5 & 6 & 7 & 8 & 0 \\
$C_{i}$ & & $\mathbf{1}$ & $\mathbf{0}$ & $\mathbf{0 . 0 0 2}$ & $\mathbf{0 . 4 9 8}$ & $\mathbf{0 . 4 8 7}$ & $\mathbf{0 . 0 1 5}$ & $\mathbf{0 . 2 5}$ & $\mathbf{0 . 4 8 5}$ \\
$d_{0 i}$ & & & 73 & 73 & 126 & 86 & 92 & 5 & 85 \\
$T_{i}$ & & 0 & 0.292 & 125.496 & 83.764 & 2.76 & 2.5 & 82.45 \\
\hline
\end{tabular}

Table 5: Route failure probabilities and additional distances for a priori tour 0-2-4-1-7-5-6-3-8-0.

\begin{tabular}{lcccccccccc}
\hline Service order & & 1 & 2 & 3 & 4 & 5 & 6 & 7 & 8 \\
priori tour & 0 & 2 & 4 & 1 & 7 & 5 & 6 & 3 & 8 & 0 \\
$C_{i}$ & & $\mathbf{1}$ & $\mathbf{0}$ & $\mathbf{0 . 0 0 2}$ & $\mathbf{0 . 4 9 8}$ & $\mathbf{0 . 4 8 7}$ & $\mathbf{0 . 0 1 5}$ & $\mathbf{0 . 2 5}$ & $\mathbf{0 . 4 8 5}$ \\
$d_{0 i}$ & & & 126 & 103 & 5 & 86 & 92 & 73 & 85 \\
$T_{i}$ & & & 0 & 0.412 & 4.98 & 83.764 & 2.76 & 36.5 & 82.45 \\
\hline
\end{tabular}

failures are shown for SC-VRPSD:

$$
\sum T_{i}=297.262
$$

The expected length of the tour (0-1-2-3-4-5-6-7-8-0) is $=607+297.262=904.262$.

Now if we take our route as 0-2-4-1-7-5-6-3-8-0 for the same sample problem, the length of 0-2-4-1-7-5-6-3-8-0 tour is $\mathrm{L}=514$ units. In Table 5, route failure probabilities and the length of additional distances stemming from the route failures are shown:

$$
\sum T_{i}=210.866
$$

The expected length of the tour (0-2-4-1-7-5-6-3-8-0) is $=514+210.866=724.866$.

As the route failure probabilities are independent from the a priori tour in case of the SC-VRPSD, the problem was regarded as an assignment problem and the non-linear 0-1 integer mathematical model was established.

Nonlinear assignment model for SC-VRPSD

$$
\begin{gathered}
\text { MIN } 2 * A+B+C, \\
A=\sum_{r=1}^{n} C_{r} * \sum_{i=1}^{n} d_{0, i} * X_{i, r}, \\
B=\sum_{i=1}^{n} d_{0, i} * X_{i, 1}+\sum_{i=1}^{n} d_{0, i} * X_{i, n}{ }^{\prime} \\
C=\sum_{\substack{i=1 \\
n}}^{n} \sum_{\substack{j=1 \\
j \neq i}}^{n} d_{i, j} * \sum_{r=1}^{n-1} X_{i, r} * X_{j, r+1},
\end{gathered}
$$


subject to

(1) $\sum_{i=1}^{n} X_{i, r}=1(r=1,2, \ldots, n)$,

(2) $\sum_{r=1}^{n} X_{i, r}=1(i=1,2, \ldots, n)$.

The symbols in the model:

0 is depot,

$i=$ customer $(i=1,2, \ldots, n)$,

$r=$ service order $(r=1,2, \ldots, n), C_{r}=$ total route failure probability in the $r$ th service order:

$$
\begin{aligned}
& X_{i, r}=1, \quad \text { if customer } i \text { is assigned to } r \text { th service order, } \\
& X_{i, r}=0, \quad \text { otherwise, }
\end{aligned}
$$

$d_{i, j}=$ the distance between customer $i$ and customer $j$.

$2 * A$ in the objective function of the model shows the cost of going back to the depot and then returning to the customer in $r$ th service order arising from the route failures, $B$ shows the distance between customers who are assigned to the first service order and to the last service order from the depot and $C$ shows the distance between customers who are successively assigned to service orders.

Whereas the constraint set (1) ensures that one customer can only be assigned to one service order, and the constraint set (2) ensures that no more than one customer can not be assigned to the same service order.

As the model is not a linear model, it is comparatively difficult to solve. The assignment problem was integrated with the TSP problem in the course of linearizing the model and thus a new linear model was established for SC-VRPSD.

Linear model for SC-VRPSD (TSP model integrated with assignment model)

$$
\begin{gathered}
\text { MIN } A+2 * B, \\
A=\sum_{i=0}^{n} \sum_{\substack{j=0 \\
j \neq i}}^{n} d_{i, j} * X_{i, j}, \\
B=\sum_{i=1}^{n} \sum_{j=1}^{n} C_{j} * d_{0, i} * Z_{i, j}
\end{gathered}
$$

subject to

$$
\begin{gathered}
\sum_{i=0}^{n} X_{i, j}=1 \quad \forall_{j}, j \neq i, \\
\sum_{j=0}^{n} X_{i, j}=1 \quad \forall_{i}, i \neq j, \\
\sum_{i=1}^{n} Z_{i, j}=1 \quad \forall_{j},(j=1,2, \ldots, n),
\end{gathered}
$$




$$
\begin{gathered}
\sum_{j=1}^{n} Z_{i, j}=1 \quad \forall_{i},(i=1,2, \ldots, n), \\
X_{0, j} \leq Z_{j, 1} \quad \forall_{j},(j=1,2, \ldots, n), \\
X_{j, 0} \leq Z_{j, n} \quad \forall_{j},(j=1,2, \ldots, n), \\
X_{0, j}+Z_{j, 1} \leq 2 \quad \forall_{j},(j=1,2, \ldots, n), \\
X_{j, 0}+Z n \leq 2 \quad \forall_{j},(j=1,2, \ldots, n), \\
\left(X_{i, j}+Z_{i, k}+\sum_{\substack{l=1 \\
l \neq k+1}}^{n} Z_{j, l}\right) \leq 2 \quad \forall_{i},(i=1,2, \ldots, n), \quad \forall_{j},(j=1,2, \ldots, n), j \neq i, \\
U(i)-U(j)+n * X_{i, j} \leq n-1 \quad \forall_{i},(i=1,2, \ldots, n), \quad \forall_{j},(j=1,2, \ldots, n) .
\end{gathered}
$$

All $X_{i, j}$ and $Z_{i, j}=0$ or 1 and all $U(i) \geq 0$ and is a set of integers.

The following symbols are used in the model:

$$
\begin{aligned}
& \begin{array}{l}
0 \text { = depot, } \\
i=\text { customer }(i=1,2, \ldots, n), \\
r=\text { service order }(r=1,2, \ldots, n), \\
C_{r}=\text { total route failure probability in the } r \text { th service order, } \\
d_{i, j}=\text { the distance between customer } i \text { and customer } j ; \\
\quad X_{i, j}=1, \quad \text { if vehicle travels directly from customer } i \text { to customer } j, \\
\quad X_{i, j}=0, \quad \text { otherwise, } \\
\quad Z_{i, j}=1, \quad \text { if customer } i \text { is assigned to } j \text { th service order, } \\
\quad Z_{i, j}=0, \quad \text { otherwise. }
\end{array}
\end{aligned}
$$

$A$ in the objective function of the model shows the cost of the TSP tour. $2 * B$ shows the cost of going back to the depot and then returning to the customers arising from route failures at the $j$ th service order. The constraint sets (4.11) and (4.12) are the constraint sets which exist in classical TSP model, whereas constraint sets (4.13) and (4.14) are present in classical assignment problem model. Constraints (4.15)-(4.19) ensure that two problems (Traveling Salesman Problem and Assignment Problem) can be solved simultaneously. Constraint (4.20) is the sub-tour elimination constraint in TSP model.

This method can be used to obtain an exact solution for the problem at a small size but for larger size problems we present here an Iterated Local Search algorithm.

\section{Iterated local search (ILS)}

ILS which is developed between meta-heuristics for difficult problems is not only an algorithm which produces effective solutions but it is also a random search method which can be easily implemented in practice. It can be referred to as the first ILS study conducted by Martin et al. [22] for Traveling Salesman Problem. Lourenço et al. [23] gained some 


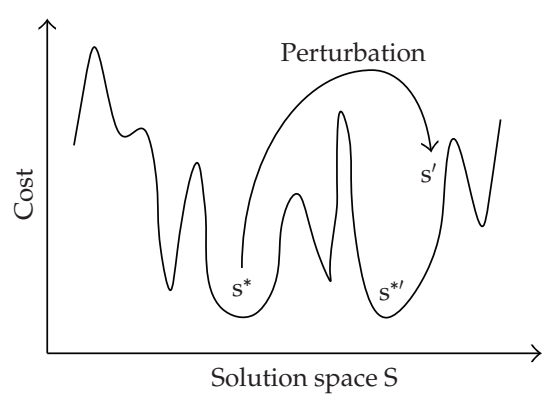

Figure 2: Perturbation phase.

information about the structure of ILS algorithm in their studies. The success of the iterated local search is not limited to TSP. Many previous studies suggest that this method was also successful in scheduling problems. Examples are Single-Machine Total Weighted Tardiness Scheduling Problem [24], flow-shop scheduling problems [25, 26], and job-shop scheduling problems [27], quadratic assignment problem [28]. For a detailed review of other applications we refer to [29].

\subsection{The general structure of ILS algorithm}

The ILS algorithm, as mentioned previously, is a random search method developed for NP-hard problems. The most important characteristic of the ILS algorithm is its ability to jump to other points of the solution space $(S)$ by masking the good characteristics of a solution which is stuck to the local optimum. This jumping action is achieved by a process called perturbation. There are four components that should be taken into consideration while applying an ILS algorithm. These are initial solution, local search, perturbation, and acceptance criterion.

\subsection{Local search}

Performance of ILS is remarkably sensitive to choice of embedded heuristic. In practice, there may be many different algorithms that can be used for the embedded heuristic. Two different local search heuristicss were used in this study in order to increase the effectiveness of the solution of the ILS algorithm. These are as follows.

\section{Two-node-exchange}

Given an a priori tour, $s_{i}=\left(s(0), s_{1}(r), \ldots, s_{n}(r), s(0)\right)$, any two nodes in the current a priori tour are exchanged. If this results in a better feasible tour the exchange is accepted. This procedure is repeated until no further improvement is achieved.

\section{2-p-opt}

Given an a priori tour, $s_{i}=\left(s(0), s_{1}(r), \ldots, s_{n}(r), s(0)\right)$, its 2-p-opt neighborhood is the set of tours obtained by reversing a section of $s$ (i.e., a set of consecutive nodes) and adjusting the arcs adjacent to the reversed section [30]. 


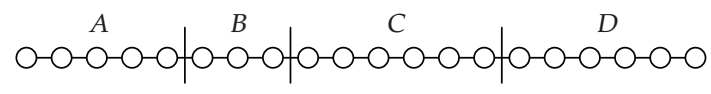

(a) Current tour before perturbation

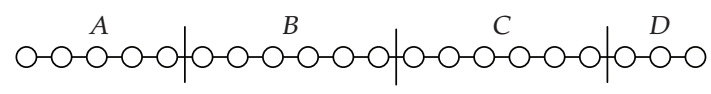

(b) New tour after perturbation

Figure 3: Double bridge move.

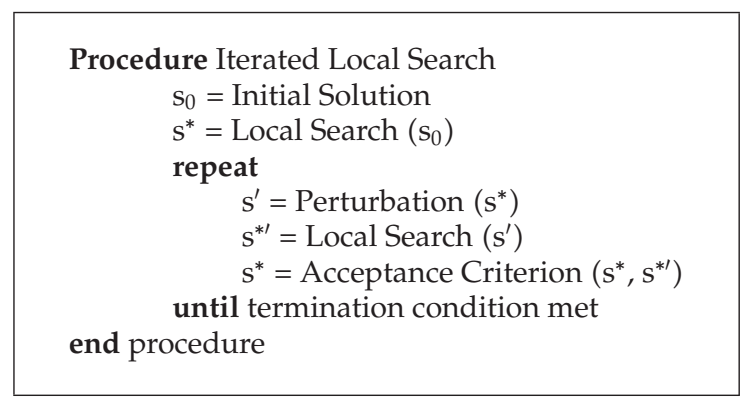

Algorithm 1: General working principles of ILS.

\subsection{Perturbation}

The objective here is to escape from local optimum by applying perturbations to the current local minimum. In Figure 2 [23], perturbation is applied the current tour $\left(\mathrm{s}^{*}\right)$ and this leads to an intermediate state $s^{\prime}$ and Local Search is applied to $s^{\prime}$ and after local search a new solution $\mathrm{s}^{* \prime}$ is reached. If $\mathrm{s}^{* \prime}$ passes an acceptance test, we accept the $\mathrm{s}^{* \prime}$ as a current tour, otherwise perturbation repeated on the $s^{*}$. Until the termination condition met, algorithm steps are repeated. Algorithm 1 shows the general working principles of ILS.

The effect of the perturbation depends on how strong the perturbation is. If the perturbation is too small, it is possible to reach the same local optimum. If the perturbation is too large, then the ILS algorithm will behave like random restart type algorithm.

The present study selected a perturbation mechanism which is effective for TSP and which is called double bridge move [23]. Double bridge move cuts the current tour at three random positions and uses a particular way of reconnecting the four remaining tour segments. Figure 3 shows the double bridge move as a perturbation mechanism.

In this study, the termination condition was established as a maximum number of iterations and the algorithm was limited to the same number of iterations for all test problems. The iteration number was taken to be the solution number applied perturbation and the maximum number of iterations was set as 1,000. In the following section, test problems and their results are discussed.

\subsection{Computational results}

The ILS algorithm was coded in Microsoft Visual C\# language and run on a PC with $1.80 \mathrm{GHz}$ CPU and 2 GB RAM. Furthermore, proposed linear mathematical model was coded using LINGO 8.0 package program. It was run for small-sized test problems up to 20 customers. 
Table 6: Comparison of ILS algorithm and the mathematical model for small-sized problems.

\begin{tabular}{|c|c|c|c|c|c|}
\hline $\begin{array}{l}\text { Problem } \\
\text { name }\end{array}$ & $\begin{array}{l}\text { Vehicle } \\
\text { capacity }\end{array}$ & $\begin{array}{c}\text { Mathematical } \\
\text { model result }\end{array}$ & $\begin{array}{l}\text { Mean CPU time } \\
\text { (sec.) for } \\
\text { mathematical } \\
\text { model }\end{array}$ & ILS result & $\begin{array}{l}\text { Mean CPU time } \\
\text { (sec.) for ILS }\end{array}$ \\
\hline \multirow{3}{*}{ Eil13 } & $Q=2.5 \mu$ & 408.99 & \multirow{3}{*}{62} & 408.994 & \multirow{3}{*}{0.77} \\
\hline & $Q=5 \mu$ & 272.364 & & 272.364 & \\
\hline & $Q=10 \mu$ & 216 & & 216 & \\
\hline \multirow{3}{*}{ Eil14 } & $Q=2.5 \mu$ & 447.536 & \multirow{3}{*}{300} & 447.536 & \multirow{3}{*}{0.82} \\
\hline & $Q=5 \mu$ & 282.344 & & 282.344 & \\
\hline & $Q=10 \mu$ & 227.988 & & 227.988 & \\
\hline \multirow{3}{*}{ Eil15 } & $Q=2.5 \mu$ & 493.852 & \multirow{3}{*}{727} & 493.852 & \multirow{3}{*}{0.88} \\
\hline & $Q=5 \mu$ & 310.268 & & 310.268 & \\
\hline & $Q=10 \mu$ & 248.048 & & 248.048 & \\
\hline \multirow{3}{*}{ Eil16 } & $Q=2.5 \mu$ & 516.768 & \multirow{3}{*}{1025} & 516.768 & \multirow{3}{*}{0.94} \\
\hline & $Q=5 \mu$ & 331.812 & & 331.812 & \\
\hline & $Q=10 \mu$ & 255.66 & & 255.66 & \\
\hline \multirow{3}{*}{ Eil17 } & $Q=2.5 \mu$ & 551.722 & \multirow{3}{*}{7111} & 551.722 & \multirow{3}{*}{1.02} \\
\hline & $Q=5 \mu$ & 350.912 & & 350.912 & \\
\hline & $Q=10 \mu$ & 259.408 & & 259.408 & \\
\hline \multirow{2}{*}{ Eil18 } & $Q=2.5 \mu$ & 577.908 & \multirow{2}{*}{7951} & 577.908 & \multirow{2}{*}{1.09} \\
\hline & $Q=5 \mu$ & 365.13 & & 365.13 & \\
\hline \multirow{2}{*}{ Eil119 } & $Q=2.5 \mu$ & 633.856 & \multirow{2}{*}{27621} & 633.856 & \multirow{2}{*}{1.19} \\
\hline & $Q=5 \mu$ & 396.706 & & 396.706 & \\
\hline Eil20 & $Q=2.5 \mu$ & 664.3 & 74421 & 664.3 & 1.32 \\
\hline
\end{tabular}

Small-sized test problems were obtained by splitting the Eil51 problem to various sizes. For example Eil20 was formed with the first 20 customers of Eil51. Similarly Eil13 includes the first 13 customers of Eil51. In all test problems, we assume that customer demands are normally distributed $\left(\xi_{i}=N(\mu, \sigma) \forall_{i} \sigma / \mu=0.2\right)$ and the first customer was accepted as the depot. At Table 6 mathematical model and ILS results were compared in terms of solution quality and CPU times to evaluate the performance of the ILS algorithm.

In all small-sized problems ILS algorithm reached fast the optimum results which were obtained by LINGO. LINGO run approximately 20.6 hours to obtain the optimum result of Eil20, however ILS reached the same result in 1.32 seconds.

Showing the validity of the solutions at small-sized test problems using ILS, the "traveling salesman" problems in the literature (Berlin52, Ei151, Eil76, Eil101, A280, KroA100, KroC100, Pr76, Lin105) were converted into SC-VRPSD problems by assuming that customer demands are normally distributed $\left(\xi_{i}=N(\mu, \sigma) \forall_{i} \sigma / \mu=0.2\right)$. The first customer was again accepted as the depot in all test problems. The results were obtained for several vehicle capacities $(\mathrm{Q}=2.5 \mu, 5 \mu, 10 \mu, 20 \mu)$. The algorithm was run 5 times for each set and the best results obtained from these 5 tests are shown in Table 7.

Test problems may be accessed online via http:/ / www.iwr.uniheidelberg.de/groups / comopt/software/TSPLIB95/. 
Table 7: Computational results for different problem sizes and vehicle capacities.

\begin{tabular}{|c|c|c|c|c|c|}
\hline \multirow{2}{*}{ Problem name } & \multirow{2}{*}{ Number of customers } & \multicolumn{4}{|c|}{ Expected tour length for various vehicle capacities } \\
\hline & & $Q=2.5 \mu$ & $Q=5 \mu$ & $Q=10 \mu$ & $Q=20 \mu$ \\
\hline Eil51 & 51 & 1669.3 & 962.35 & 632.01 & 490.512 \\
\hline Berlin52 & 52 & 27700.498 & 16385.908 & 10865.498 & 8557.894 \\
\hline Eil76 & 76 & 2812.25 & 1550.808 & 943.856 & 650.714 \\
\hline $\operatorname{Pr} 76$ & 76 & 785557.476 & 408383.298 & 238931.054 & 158792.47 \\
\hline KroA100 & 100 & 152966.996 & 80303.686 & 47105.406 & 30644.136 \\
\hline KroC100 & 100 & 171777.932 & 88675.256 & 50903.832 & 32812.934 \\
\hline Eil101 & 101 & 3385.564 & 1861.12 & 1143.17 & 815.756 \\
\hline Lin105 & 105 & 178862.26 & 88474.332 & 48050.174 & 28946.026 \\
\hline A 280 & 280 & 49040.85 & 23590.382 & 12443.506 & 7156.779 \\
\hline
\end{tabular}

\section{Conclusion}

The present study defined a special case for the vehicle routing problem with stochastic demands (SC-VRPSD) where customer demands are normally distributed. A new mathematical model was proposed for the calculation of the length of tour for SC-VRPSD. Proposed model is based on the integration of the "Traveling Salesman Problem" (TSP) and the Assignment Problem. However, the Iterated Local Search algorithm (ILS) was used in order to reach an appropriate solution because the linear model could not produce solutions in polynomial time for large-scale problems. Test problems were obtained from the conversion of well-known TSP problems in the literature to SC-VRPSD. The results obtained for the test problems may be used for comparison purposes for further research. The results may be improved by further refinement of the algorithms and incorporation of suggestions from other researchers.

\section{References}

[1] G. Laporte and I. H. Osman, "Routing problems: a bibliography," Annals of Operations Research, vol. 61, no. 1, pp. 227-262, 1995.

[2] P. Toth and D. Vigo, "Models, relaxations and exact approaches for the capacitated vehicle routing problem," Discrete Applied Mathematics, vol. 123, no. 1-3, pp. 487-512, 2002.

[3] G. Laporte, M. Gendreau, J.-Y. Potvin, and F. Semet, "Classical and modern heuristics for the vehicle routing problem," International Transactions in Operational Research, vol. 7, no. 4-5, pp. 285-300, 2000.

[4] C. D. Tarantilis, G. Ioannou, and G. Prastacos, "Advanced vehicle routing algorithms for complex operations management problems," Journal of Food Engineering, vol. 70, no. 3, pp. 455-471, 2005.

[5] M. Gendreau, G. Laporte, and R. Séguin, "Stochastic vehicle routing," European Journal of Operational Research, vol. 88, no. 1, pp. 3-12, 1996.

[6] M. Dror, M. Ball, and B. Golden, "A computational comparison of algorithms for the inventory routing problem," Annals of Operations Research, vol. 4, no. 1-4, pp. 3-23, 1985.

[7] V. Lambert, G. Laporte, and F. Louveaux, "Designing collection routes through bank branches," Computers E Operations Research, vol. 20, no. 7, pp. 783-791, 1993.

[8] R. C. Larson, "Transporting sludge to the 106-mile site: an inventory/routing model for fleet sizing and logistics system design," Transportation Science, vol. 22, no. 3, pp. 186-198, 1988.

[9] K. C. Tan, C. Y. Cheong, and C. K. Goh, "Solving multiobjective vehicle routing problem with stochastic demand via evolutionary computation," European Journal of Operational Research, vol. 177, no. 2, pp. 813-839, 2007.

[10] D. Bertsimas, P. Chervi, and M. Peterson, "Computational approaches to stochastic vehicle routing problems," Transportation Science, vol. 29, no. 4, pp. 342-352, 1995. 
[11] W.-H. Yang, K. Mathur, and R. H. Ballou, "Stochastic vehicle routing problem with restocking," Transportation Science, vol. 34, no. 1, pp. 99-112, 2000.

[12] L. Bianchi, M. Birattari, M. Chiarandini, et al., "Hybrid metaheuristics for the vehicle routing problem with stochastic demands," Journal of Mathematical Modelling and Algorithms, vol. 5, no. 1, pp. 91-110, 2006.

[13] N. Secomandi, "Comparing neuro-dynamic programming algorithms for the vehicle routing problem with stochastic demands," Computers E Operations Research, vol. 27, no. 11-12, pp. 1201-1225, 2000.

[14] N. Secomandi, "A rollout policy for the vehicle routing problem with stochastic demands," Operations Research, vol. 49, no. 5, pp. 796-802, 2001.

[15] M. Gendreau, G. Laporte, and R. Séguin, "An exact algorithm for the vehicle routing problem with stochastic demands and customers," Transportation Sciences, vol. 29, no. 2, pp. 143-155, 1995.

[16] M. Gendreau, G. Laporte, and R. Séguin, "A tabu search heuristic for the vehicle routing problem with stochastic demands and customers," Operations Research, vol. 44, no. 3, pp. 469-477, 1996.

[17] D. Teodorović and G. Pavković, "A simulated annealing technique approach to the vehicle routing problem in the case of stochastic demand," Transportation Planning and Technology, vol. 16, no. 4, pp. 261-273, 1992.

[18] S. K. Isleyen and O. F. Baykoc, "An efficiently novel model for vehicle routing problems with stochastic demands," Asia-Pacific Journal of Operational Research. In press.

[19] M. Dror and P. Trudeau, "Stochastic vehicle routing with modified savings algorithm," European Journal of Operational Research, vol. 23, no. 2, pp. 228-235, 1986.

[20] D. J. Bertsimas and D. Simchi-Levi, "A new generation of vehicle routing research: robust algorithms, addressing uncertainty," Operations Research, vol. 44, no. 2, pp. 286-304, 1996.

[21] A. S. Kenyon and D. P. Morton, "A survey on stochastic location and routing problems," Central European Journal of Operations Research, vol. 9, no. 4, pp. 277-328, 2001.

[22] O. Martin, S. W. Otto, and E. W. Felten, "Large-step Markov chains for the TSP incorporating local search heuristics," Operations Research Letters, vol. 11, no. 4, pp. 219-224, 1992.

[23] H. R. Lourenço, O. C. Martin, and T. Stützle, "A beginner's introduction to iterated local search," in Proceedings of the 4th Metaheuristics International Conference (MIC '01), vol. 2, pp. 545-550, Porto, Portugal, July 2001.

[24] R. K. Congram, C. N. Potts, and S. L. van de Velde, “An iterated dynasearch algorithm for the singlemachine total weighted tardiness scheduling problem," INFORMS Journal on Computing, vol. 14, no. 1, pp. 52-67, 2002.

[25] T. Stützle, "Applying iterated local search to the permutation flow shop problem," Tech. Rep. AIDA98-04, FG Intellektik, TU Darmstadt, Darmstadt, Germany, 1998.

[26] Y. Yang, S. Kreipl, and M. Pinedo, "Heuristics for minimizing total weighted tardiness in flexible flow shops," Journal of Scheduling, vol. 3, no. 2, pp. 89-108, 2000.

[27] E. Balas and A. Vazacopoulos, "Guided local search with shifting bottleneck for job shop scheduling," Management Science, vol. 44, no. 2, pp. 262-275, 1998.

[28] T. Stützle, "Iterated local search for the quadratic assignment problem," European Journal of Operational Research, vol. 174, no. 3, pp. 1519-1539, 2006.

[29] H. R. Lourenço, O. C. Martin, and T. Stützle, "Iterated local search," in Handbook of Metaheuristics, vol. 57 of International Series in Operations Research E Management Science, pp. 321-353, Kluwer Academic Publishers, Boston, Mass, USA, 2003.

[30] L. Bianchi, J. Knowles, and N. Bowler, "Local search for the probabilistic traveling salesman problem: correction to the 2-p-opt and 1-shift algorithms," European Journal of Operational Research, vol. 162, no. 1, pp. 206-219, 2005. 


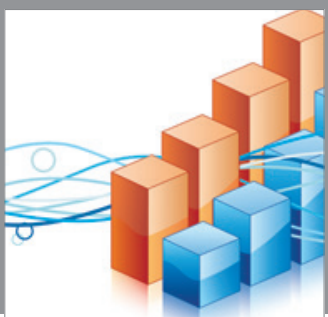

Advances in

Operations Research

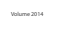

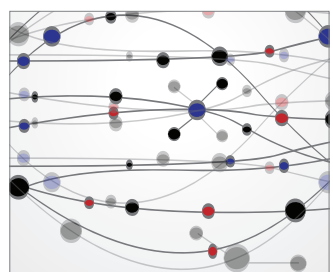

\section{The Scientific} World Journal
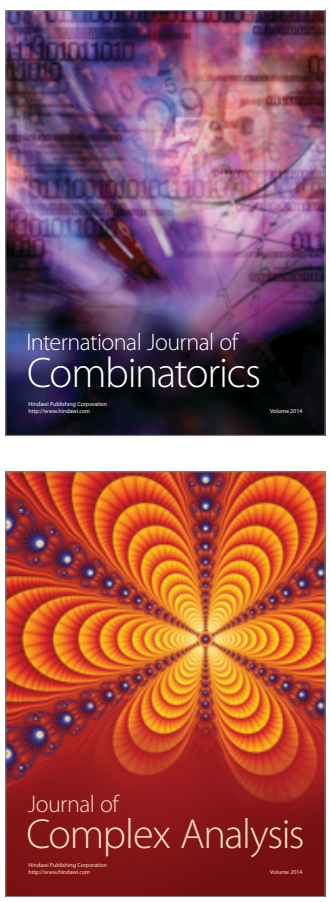

International Journal of

Mathematics and

Mathematical

Sciences
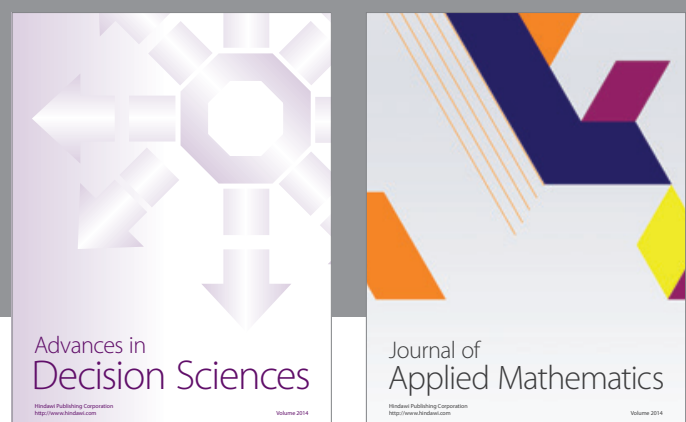

Journal of

Applied Mathematics
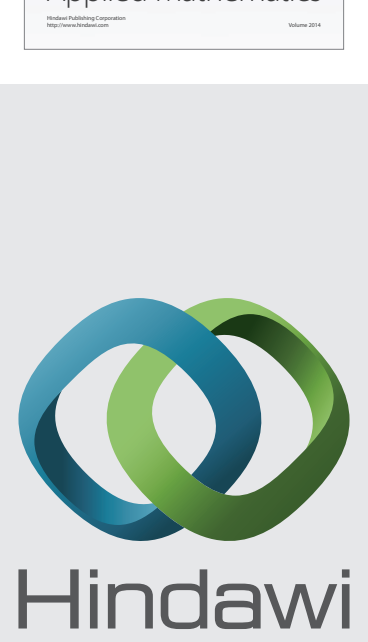

Submit your manuscripts at http://www.hindawi.com
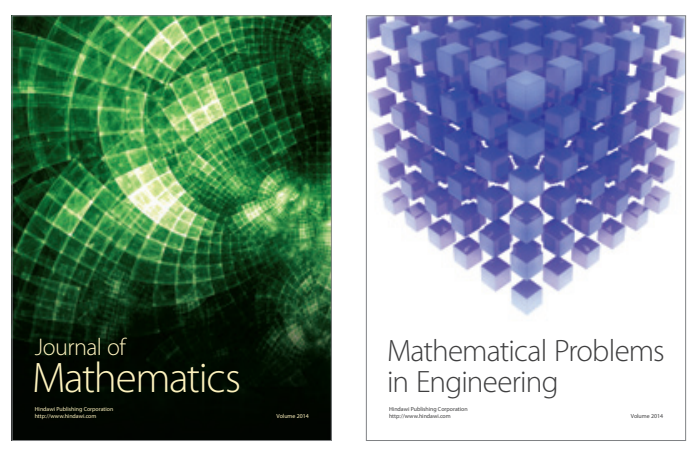

Mathematical Problems in Engineering
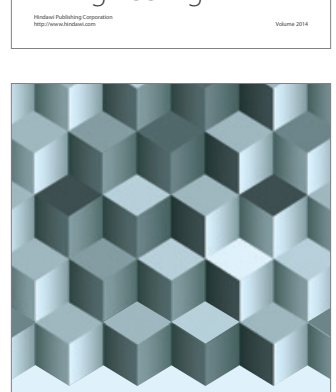

Journal of

Function Spaces
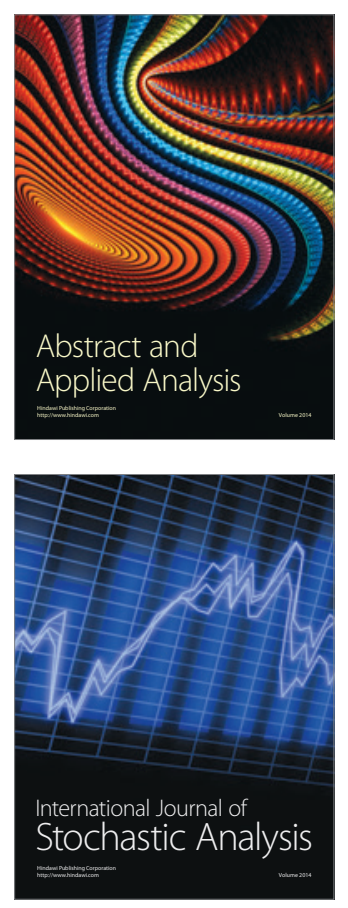

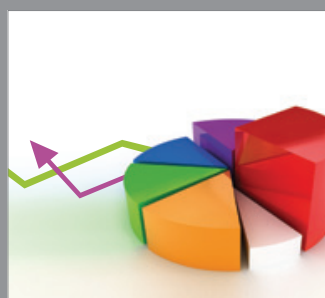

ournal of

Probability and Statistics

Promensencen
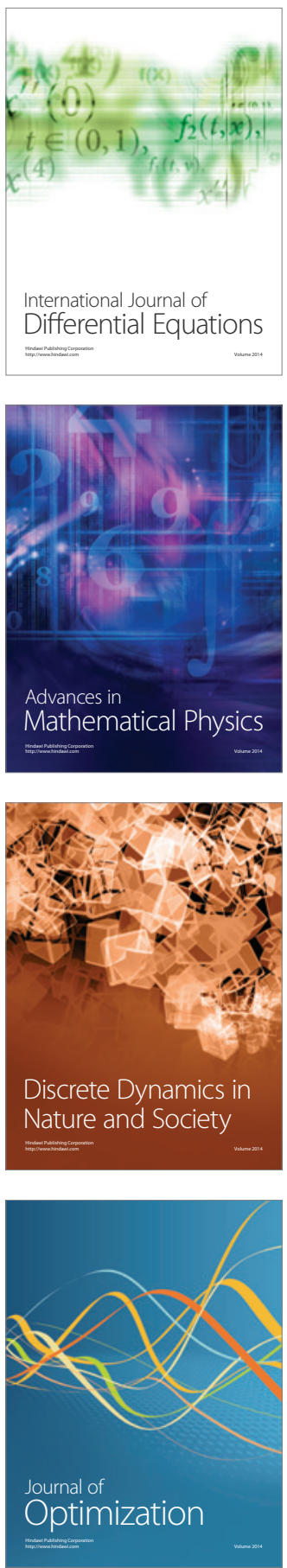